\title{
Using the clinical chemistry score in the emergency department to detect adverse cardiac events: a diagnostic accuracy study
}

\author{
Peter A. Kavsak PhD, Joshua O. Cerasuolo MSc, Dennis T. Ko MD MSc, Jinhui Ma PhD, \\ Jonathan Sherbino MD, Shawn E. Mondoux MD, Natasha Clayton CRA RA, Stephen A. Hill PhD, \\ Matthew McQueen MBChB PhD, Lauren E. Griffith PhD, Shamir R. Mehta MD MSc, \\ Richard Perez MSc, Hsien Seow PhD, P.J. Devereaux MD PhD, Andrew Worster MD MSc
}

\section{Abstract}

Background: The ability to rule out or in a major adverse cardiac event (MACE) in patients with suspected acute coronary syndrome at emergency department (ED) presentation would be beneficial to patient care and the health care system. The clinical chemistry score (CCS) was evaluated in this context.

Methods: This diagnostic accuracy study evaluated 2 different ED cohorts with suspected acute coronary syndrome. For patients in cohort 1, who presented to the ED of 3 hospitals in Hamilton, Ontario, between May and August 2013, retrospective measurements were taken using the Ortho Clinical Diagnostics high-sensitivity cardiac troponin I (hs-cTnl) assay; for patients in cohort 2, who presented to the ED of the same 3 hospitals in Hamilton between November 2012 and February 2013, an ED cardiac presentation blood test panel was performed with the Abbott Diagnostics hs-cTnl assay. The sensitivity and specificity of the CCS (cut-offs of $\geq 1$ and 5 ) and hs-cTnl alone (published cut-offs) were compared for MACE (composite of death, myocardial infarction, unstable angina, revascularization) at 30 days for both cohorts and at 90 days for cohort 2 .

Results: The incidence of MACE at 30 days was higher in cohort $1(n=1058)(19.4 \%, 95 \%$ confidence interval [Cl] $16.8 \%-22.2 \%)$ than in cohort $2(n=5974)(14.6 \%, 95 \% \mathrm{Cl} 13.6 \%-15.6 \%)$. In cohort 1 , a CCS of 1 or above yielded a sensitivity of $99.5 \%(95 \% \mathrm{Cl} 97.3 \%-99.9 \%)$. The sensitivity with an Ortho hs-cTnl cut-off of $1 \mathrm{ng} / \mathrm{L}$ or above was $91.2 \%$ (95\% Cl $86.5 \%-95.7 \%)$. The specificity of a CCS of 5 ( $97.8 \%$, 95\% Cl 96.5\%-98.7\%) was higher than when the overall 99th-percentile cut-off for the Ortho hs-cTnl assay (> 11 ng/L; 90.1\%, 95\% Cl $87.9 \%-92.0 \%$ ) was used. A similar pattern was observed in cohort 2 at 30 days and persisted at 90 days with the Abbott hs-cTnl assay.

Interpretation: The CCS derived with 2 different hs-cTnl assays and ED populations yielded higher sensitivity and specificity estimates for MACE than hs-cTnl alone. An intervention study is needed to evaluate the impact of the CCS at both the patient and hospital levels. Trial registration: ClinicalTrials.gov, no. NCT01994577

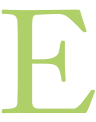

vidence and guidelines have indicated that an undetectable or low concentration of high-sensitivity cardiac troponin (hs-cTn) at emergency department (ED) presentation may be a suitable criterion in certain patients for ruling out myocardial infarction (MI) and possibly discharging patients home. ${ }^{1-4}$ The basis for this is the high clinical sensitivity and negative predictive value (NPV) that can be attained when an hs-cTn cut-off close to $5 \mathrm{ng} / \mathrm{L}$ is used. ${ }^{2,3,5,6}$ However, the usefulness of an undetectable or low hs-cTn concentration in ruling out MI does not necessarily mean that it can also be used to predict whether a patient is at low risk for a subsequent cardiovascular event.

An international survey of ED physicians indicated that an acceptable miss rate of major adverse cardiac events (MACE) within 30 days would be $1 \%$ or less. ${ }^{7}$ Accordingly, the sensitivity required for MACE at 30 days should be at least $99 \%$ to achieve this threshold and to allow discharge and cessation of
Competing interests: Peter Kavsak has received grants from Ortho Clinical Diagnostics and Abbott Laboratories for aspects of the present study. Outside this study, Peter Kavsak has received grants from Abbott Laboratories, Beckman Coulter, Ortho Clinical Diagnostics, Roche Diagnostics and Siemens Healthcare Diagnostics; personal fees from Abbott Laboratories, Beckman Coulter, Randox, Roche Diagnostics and Siemens Healthcare Diagnostics; and nonfinancial support from Randox and Siemens Healthcare Diagnostics. McMaster University has filed patents with Peter Kavsak and Andrew Worster listed as inventors in the acute cardiovascular biomarker field. In particular, a patent related to the data in this study has been filed, for a laboratory score for risk stratification for patients with possible cardiac injury. P.J. Devereaux has received grants from Abbott Diagnostics, Boehringer Ingelheim, Philips Healthcare, Roche Diagnostics and Siemens and product support from Philips Healthcare, outside the present study. No other competing interests were declared.

This article has been peer reviewed.

Correspondence to: Peter Kavsak, kavsakp@mcmaster.ca CMAJ Open 2020. DOI:10.9778/cmajo.20200047 
investigations in patients presenting with possible acute coronary syndrome (ACS) to the ED. ${ }^{7}$ Therefore, uncertainty exists about whether a single low hs-cTn cut-off at presentation provides sufficient sensitivity to exclude 30-day MACE, with the American College of Emergency Physicians issuing the following statement in its latest clinical policy document: "a single high-sensitivity troponin may not have adequate sensitivity for MACE." A recent study in patients with suspected ACS in the United States that assessed death, MI and myocardial revascularization within 30 days found that 2 measurements of hs-cTn (at 0 and $3 \mathrm{~h}$ ) below $6 \mathrm{ng} / \mathrm{L}$ achieved an NPV of $99.3 \%$, but no data were provided for the sensitivity and NPV of the measurement (at $0 \mathrm{~h}$ ) alone using this low cut-off. ${ }^{9}$

We have previously demonstrated in over 4000 patients enrolled in clinical studies assessing hs-cTn in those with suspected ACS that a simple clinical chemistry score (CCS) that includes measurements of glucose, creatinine (for estimated glomerular filtration rate, eGFR) and hs-cTn provided sensitivity $(\geq 99.9 \%)$ for 30 -day $\mathrm{MI}$ or death that was superior to that of the first 2 hs-cTn assays approved by Health Canada, namely Roche high-sensitivity cardiac troponin $\mathrm{T}$ (hs-c TnT; sensitivity 98.2\%) and Abbott high-sensitivity cardiac troponin I (hs-cTnI; sensitivity 96.6\%). ${ }^{10}$ Since that publication, 3 additional companies (Beckman Coulter, Siemens Healthcare Diagnostics and Ortho Clinical Diagnostics) have obtained regulatory approval for hs-cTnI assays from Health Canada. ${ }^{11-14}$

There is no standardization between hs-cTnI assays, so different hs-c TnI assays may give very different results for the same sample (cTnT and cTnI are different proteins, so the corresponding assays will produce different results). ${ }^{15,16}$ For example, for a given patient, the same lithium heparin plasma material measured on the Abbott Architect 11000 (hs-cTnI), Siemens Dimension EXL (hs-cTnI) and Roche Cobas (hs-cTnT) instruments yielded mean hs-cTn results of $4.4 \mathrm{ng} / \mathrm{L}, 7.2 \mathrm{ng} / \mathrm{L}$ and $26.8 \mathrm{ng} / \mathrm{L}$, respectively. ${ }^{16}$ (Diagnostic companies are discontinuing non-hs-cTn assays, so the standard laboratory test for evaluating myocardial injury will be the hs-cTn assays. ${ }^{17}$ ) There are also interferences that may affect some but not all assays, and thus it is important that users know the manufacturer of the hs-cTn assay they are using so that they can interpret results appropriately. ${ }^{18,19}$

The goal of this study was to determine if the CCS, calculated using results from different hs-cTnI assays, could achieve the same diagnostic performance ( $\geq 99 \%$ sensitivity, $\geq 90 \%$ specificity $)^{10,20}$ as that observed in the clinical study setting and to determine if it was superior to hs-cTnI alone, for 30-day MACE, in a general ED population investigated for possible acute cardiac injury.

\section{Methods}

\section{Study design and patient cohorts}

We conducted a diagnostic accuracy study of the CCS using 2 ED observational cohorts with parallel hs-cTn and conventional troponin measurements performed; hs-cTn results remained unreported to the treating ED physician (i.e., clinical staff were blinded to hs-c'Tn results). The earliest blood draw in the ED that included all laboratory measurements was used in both studies. This was accomplished in accordance with the Standards for the Reporting of Diagnostic Accuracy Studies guideline.

Cohort 1 was the Canadian cohort used in the initial publication on the CCS. ${ }^{10}$ Briefly, that publication reported the results of an observational cohort study (NCT01994577) that enrolled patients presenting to the ED of 3 hospitals (Hamilton General Hospital, Juravinski Hospital and St. Joseph's Healthcare Hamilton) in Hamilton, Ontario, between May 2013 and August 2013. Eligible patients were 18 years of age and older, had not been transferred from a different hospital and had a cardiac troponin measurement ordered by an ED physician. Patients were excluded if their symptoms were non-ACS (i.e., symptoms that did not belong to the following group of symptoms: chest pain or discomfort; pain or discomfort in 1 or both arms; pain or discomfort in the jaw, neck or back; pain or discomfort in the abdomen; shortness of breath; feeling dizzy or lightheaded; nausea or vomiting or both; diaphoresis; palpitations); ${ }^{4}$ or if they had ST-segment elevation MI (STEMI) at presentation; or if they had chest trauma, cardiac surgery or manipulation within 30 days of presentation; or if they had a MI (STEMI or non-STEMI) or pulmonary embolus confirmed within the previous month; or if they had known active cancer or noncardiac fatal illness; or if they had sepsis; or if they had ventricular fibrillation or sustained ventricular tachycardia. ${ }^{1021-23}$ Ethylenediaminetetraacetic acid (EDTA) plasma samples were collected and stored below $-70^{\circ} \mathrm{C}$. Further selection for the cohort was dependent of the availability of a presentation sample with sufficient volume to obtain a result for the Ortho Vitros 3600 hs-cTnI assay (Ortho Clinical Diagnostics) (Figure 1).

Cohort 2 was also from an observational study in which consecutive patients (aged 12-104 yr) who visited the ED at the same 3 hospitals (Hamilton General Hospital, Juravinski Hospital and St. Joseph's Healthcare Hamilton) in Hamilton between November 2012 and February 2013 and had a cardiac troponin I measurement ordered, also had an hs-cTnI measurement performed on the same tube (Abbott Architect i2000 hs-cTnI measured but not reported). ${ }^{20,24}$ Patients were excluded if data for their age or sex were missing in the Ontario Registered Persons Database, if they were not an Ontario resident or eligible for the Ontario Health Insurance Plan (OHIP) or if glucose and creatinine results at presentation were not available for them (note that in Hamilton there is an ED cardiac laboratory order set that includes glucose, creatinine and other blood tests to be ordered in addition to a troponin test) (Figure 1).

\section{Health outcomes}

The outcome for this analysis was MACE that occurred after the blood workup at ED presentation within 30 days in both cohorts and 90 days in cohort 2. MACE was defined as the composite outcome of MI, unstable angina, revascularization (e.g., percutaneous coronary intervention or coronary artery bypass grafting) or all-cause death. In cohort 1 , an emergency 


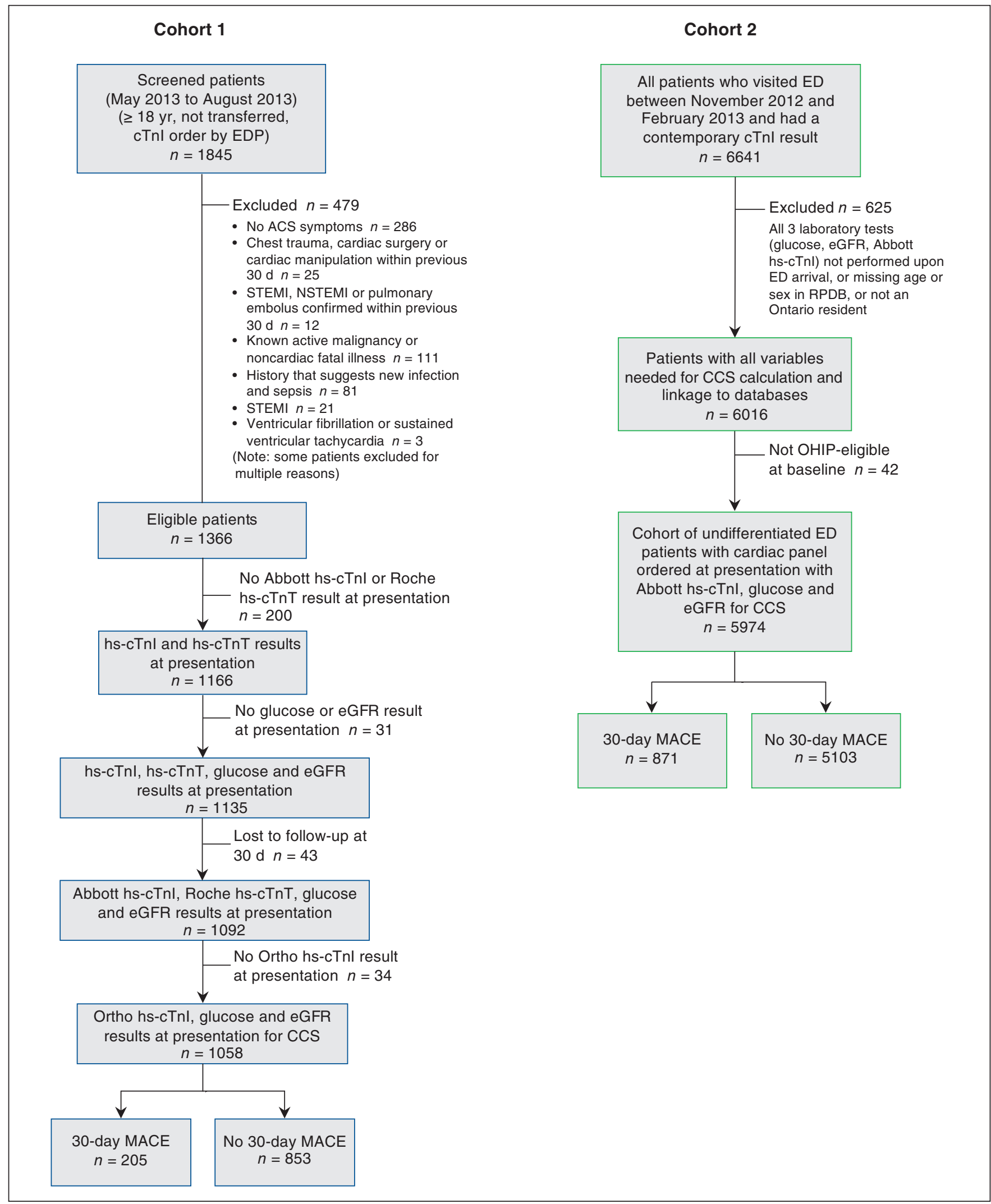

Figure 1: Flow diagram of the 2 different ED cohorts in this study. ACS = acute coronary syndrome, CCS = clinical chemistry score, hs-cTnl = highsensitivity cardiac troponin I, hs-cTnT = high-sensitivity cardiac troponin T, ED = emergency department, EDP = emergency department physician, eGFR = estimated glomerular filtration rate, MACE = major adverse cardiac event, NSTEMI = non-ST-segment elevation myocardial infarction, OHIP = Ontario Health Insurance Plan, RPDB = Registered Persons Database, STEMI = ST-segment elevation myocardial infarction. 
physician led an adjudication panel; the outcomes were independently adjudicated by at least 2 members and disagreements not resolved by consensus were referred to a third blinded reviewer ${ }^{10}$ (Appendix 1, available at www.cmajopen.ca /content/8/4/E676/suppl/DC1).

In cohort 2, adjudication was not performed; rather, administrative and clinical databases linked at ICES (Toronto) were used via unique encrypted patient identifiers to obtain information on medical history and outcomes (all-cause death; or hospital admission from MI; or unstable angina; or percutaneous coronary intervention or coronary artery bypass grafting). ${ }^{20,25}$ Briefly, the Ontario Registered Persons Database contained all information on patient demographic characteristics and death date. All inpatient hospital discharges and same-day surgeries were captured in the Canadian Institute for Health Information's Discharge Abstract Database and Same Day Surgery Database, respectively. The OHIP database captured all physician billings and outpatient visits (see Appendix 1 for additional details regarding health outcomes). Per ICES standard operating procedures, data for counts or group sizes smaller than 6 in cohort 2 were suppressed for privacy reasons and cannot be reported..$^{20,25}$

\section{Laboratory methods and cut-offs}

The hs-cTnI assay selected for measurement in cohort 1 was from Ortho Clinical Diagnostics. Compared with other companies' hs-c'TnI assays, the Ortho hs-cTnI assay generally yields the lowest $\mathrm{c} T \mathrm{TI}$ concentrations. ${ }^{12-14}$ Testing performed on patient pool plasma material in citrate phosphate dextrose (stored for quality control purposes) used to validate hs-c TnI assays supports this finding. Published mean concentrations on this material were as follows: Beckman Access hs-cTnI, 3.4 ng/L; Abbott Architect i1000 hs-cTnI, 5.1 ng/L; Siemens Advia Centaur hs-cTnI, $5.0 \mathrm{ng} / \mathrm{L}$; and Siemens Dimension EXL hs-cTnI, $8.6 \mathrm{ng} / \mathrm{L} ; 11,13,26,27$ measurement of this material by the Ortho Vitros 3600 hs-cTnI assay yielded the lowest concentration (Ortho hs-cTnI mean $1.1 \mathrm{ng} / \mathrm{L}$ ). ${ }^{14}$ The suitability of frozen samples (stored below $-70^{\circ} \mathrm{C}$ for over $15 \mathrm{yr}$ ) has also been demonstrated for the Ortho hs-cTnI assay, consistent with other assays. ${ }^{14,28}$

To further demonstrate differences between the various hscTnI assays, 10 different EDTA plasma samples were measured on the Abbott Architect i1000 (hs-cTnI range $4.7-43 \mathrm{ng} / \mathrm{L}$, with EDTA being the only sample type approved by the United States Food and Drug Administration for the Abbott hs-cTnI assay on the Architect analyzer) ${ }^{29}$ and on the Siemens Advia Centaur, the Beckman Access and the Ortho Vitros 3600 analyzers. All 3 hs-cTnI assays yielded lower results than the Abbott assay in EDTA plasma, with Siemens reporting on average $26 \%$ lower concentrations, Beckman reporting on average $30 \%$ lower concentrations and Ortho reporting on average $65 \%$ lower concentrations. The hs-cTnI assays also differ in the targeted cTnI epitopes, the mode in which the signal is generated, as well as the number of antibodies used in the respective hs-cTn assays. In this regard, the latest assay approved, the Ortho hs-cTnI assay, uses 3 antibodies, with data indicating less interference with endogenous antibodies. ${ }^{19}$ The specimen types presently listed in package inserts approved by Health Canada are as follows. For Abbott, the specimen types are lithium heparin with and without separator, $\mathrm{K}_{2}$ EDTA, $\mathrm{K}_{3}$ EDTA, serum with and without separator, and serum with thrombin-based clot activator. For Ortho, the specimen types are serum and plasma (lithium heparin).

In addition to results for conventional cardiac troponin I, which were reported to the treating ED physician for the standard of care diagnosis, Abbott hs-cTnI testing was also performed for cohort 2 but the results remained unreported (i.e., clinical samples were measured twice: once for the contemporary assay, which was reported to clinicians, and once with the hs-cTnI assay, with results not reported but collected for study purposes). ${ }^{20,24}$

All cardiac troponin testing performed in both cohort 1 and cohort 2 was consistent with laboratory quality recommendations published in 2018. ${ }^{15}$ Glucose and creatinine measurements were obtained according to standard acceptable laboratory methods (Abbott Architect chemistry analyzer), with the CKD-EPI equation used to derive the estimated glomerular filtration rate $(\mathrm{eGFR})$ : eGFR $=141 \times \min (\mathrm{Scr} / \kappa, 1)^{\alpha} \times$ $\max (\mathrm{Scr} / \kappa, 1)^{-1.209} \times 0.993^{\mathrm{Age}} \times 1.018$ (if female) $\times 1.159$ (if Black), where Scr is serum creatinine, $\kappa$ is 0.7 for women and 0.9 for men, $\alpha$ is -0.329 for women and -0.411 for men, $\min$ indicates the minimum of $S \mathrm{cr} / \mathrm{K}$ or 1 , and max indicates the maximum of $S \mathrm{cr} / \kappa$ or $1^{30}$ (this derivation was done only after the studies were completed; in other words, the eGFR result calculated by this equation was not clinically reported during the enrolment).

The cut-offs selected were a CCS of 1 or above and an hscTnI concentration of $5 \mathrm{ng} / \mathrm{L}$ or above alone, as both of these cut-offs have been reported in the literature for ruling out MI, with $5 \mathrm{ng} / \mathrm{L}$ proposed as a common cut-off for hs-c Tn assays. ${ }^{3,6,10,31} \mathrm{~A}$ second cut-off for the Ortho hs-cTnI assay of $1 \mathrm{ng} / \mathrm{L}$ or above was also evaluated as this cut-off has recently been reported for the European Society of Cardiology 0-hour and 1-hour algorithm. ${ }^{32}$ Briefly, the CCS calculation includes glucose, eGFR and hs-cTnI with the cut-offs selected on the basis of accepted normal levels and low-risk clinical cut points and has a range of $0-5$ points. ${ }^{10}$ For example, patients with an eGFR of $90 \mathrm{~mL} / \mathrm{min}$ per $1.73 \mathrm{~m}^{2}$ or above, a glucose concentration less than $5.6 \mathrm{mmol} / \mathrm{L}$ and an hs-cTnI concentration less than $4 \mathrm{ng} / \mathrm{L}$ (a normal concentration by all hs-cTnI assays) ${ }^{33}$ would have a CCS less than 1, and this score would represent biochemically healthy individuals. ${ }^{10}$ In contrast, patients who have a glucose concentration of $5.6 \mathrm{mmol} / \mathrm{L}$ or above, an eGFR less than $90 \mathrm{~mL} / \mathrm{min}$ per $1.73 \mathrm{~m}^{2}$ and an hs-cTnI concentration above $30 \mathrm{ng} / \mathrm{L}^{10}$ would have a $\mathrm{CCS}$ of 5 .

\section{Statistical analysis}

The diagnostic test parameters (sensitivity, specificity, NPV, positive predictive value [PPV] and likelihood ratios with $95 \%$ confidence intervals [CIs]) were calculated using a CCS of 1 or above and an hs-c TnI concentration of $5 \mathrm{ng} / \mathrm{L}$ or above (for low risk) and a CCS of 5 and an hs-cTnI concentration above the 99th percentile upper reference limit (for high risk) as the cut-offs in both cohorts. Additionally, in cohort 1, a 
cut-off with an hs-cTnI concentration of $1 \mathrm{ng} / \mathrm{L}$ was also evaluated. Common variables between the 2 cohorts were calculated (mean, standard deviation or percentages).

Clinical significance of the hs-cTnI test would be ascertained if the point estimate of the sensitivity was $99 \%$ or higher and the specificity was $90 \%$ or higher. ${ }^{7,8,10,20}$ As indicated earlier, in cohort 2, data for counts less than 6 were not reported to protect privacy per ICES protocols. ${ }^{20,25}$ Accordingly, if counts less than 6 were obtained at 30 days, an additional analysis was performed at 90 days to obtain numbers to calculate the $95 \%$ CIs. Statistical analyses were performed using SAS version 9.1.3 software (SAS Institute Inc.) and MedCalc version 19.4.0 (MedCalc Software Ltd.) for the sensitivity, specificity, predictive values, likelihood ratios and CIs.

\section{Ethics approval}

The studies (cohort 1 and cohort 2 observational studies) were approved by the Hamilton Integrated Research Ethics Board.

\section{Results}

In both cohort $1(n=1058)$ and cohort $2(n=5974)$, the average age of patients exceeded 65 years $(66.5$ and $67.7 \mathrm{yr}$, respectively) with over half of the population being women $(52.7 \%$ and $50.6 \%$, respectively) (Table 1). The hs-cTnI concentration at presentation was normal (i.e., below the 99th percentile cut-off, which is designated as the upper reference limit) in

Table 1: Characteristics of the 2 study cohorts

\begin{tabular}{|c|c|c|}
\hline \multirow[b]{2}{*}{ Characteristic } & \multicolumn{2}{|c|}{ No. $(\%)$ of patients* } \\
\hline & $\begin{array}{l}\text { Cohort } 1 \\
n=1058\end{array}$ & $\begin{array}{l}\text { Cohort } 2 \\
n=5974\end{array}$ \\
\hline Age, yr, mean \pm SD & $67 \pm 17$ & $68 \pm 17$ \\
\hline Female sex & $558(52.7)$ & $3025(50.6)$ \\
\hline Hypertension & 744 (70.3) & 4045 (67.7) \\
\hline Diabetes mellitus & $312(29.4)$ & $1961(32.8)$ \\
\hline $\begin{array}{l}\text { History of myocardial } \\
\text { infarction }\end{array}$ & $378(35.7)$ & $674(11.3)$ \\
\hline History of $\mathrm{PCl}$ or $\mathrm{CABG}$ & 236 (22.3) & $453(7.6)$ \\
\hline $\begin{array}{l}\text { History of peripheral vascular } \\
\text { disease }\end{array}$ & $75(7.1)$ & $742(12.4)$ \\
\hline $\begin{array}{l}\text { Glucose concentration, } \\
\mathrm{mmol} / \mathrm{L}, \text { mean } \pm \mathrm{SD}\end{array}$ & $7.4 \pm 3.6$ & $7.6 \pm 4.4$ \\
\hline $\begin{array}{l}\text { eGFR, } \mathrm{mL} / \mathrm{min} \text { per } 1.73 \mathrm{~m}^{2} \\
\text { mean } \pm \mathrm{SD}\end{array}$ & $72 \pm 27$ & $69 \pm 27$ \\
\hline $\begin{array}{l}\text { hs-cTnl concentration at } \\
\text { presentation < 99th } \\
\text { percentile cut-off } †\end{array}$ & $842(79.6)$ & $4698(78.6)$ \\
\hline 30-day MACE & 205 (19.4) & $871(14.6)$ \\
\hline \multicolumn{3}{|c|}{$\begin{array}{l}\text { Note: } \mathrm{CABG}=\text { coronary artery bypass grafting, eGFR = estimated glomerular } \\
\text { filtration rate, hs-CTNI = high-sensitivity cardiac troponin I, MACE = major adverse } \\
\text { cardiac event, } \mathrm{PCl}=\text { percutaneous coronary intervention, } \mathrm{SD}=\text { standard deviation. } \\
\text { *Unless indicated otherwise. } \\
\text { t99th percentile upper reference limit is } 11 \mathrm{ng} / \mathrm{L} \text { for Ortho hs-cTnl assay and } \\
26 \mathrm{ng} / \mathrm{L} \text { for Abbott hs-cTnl assay. }\end{array}$} \\
\hline
\end{tabular}

over three-quarters of the population $(79.6 \%$ in cohort 1 and $78.6 \%$ in cohort 2). The MACE incidence at 30 days was higher in cohort $1(19.4 \%, 95 \%$ CI $16.8 \%-22.2 \%)$ than in cohort $2(14.6 \%, 95 \%$ CI $13.6 \%-15.6 \%)(p<0.01)$.

In cohort 1 , there were 110 patients with a CCS less than 1 (10.4\% of the sample); 1 ( $0.9 \%)$ of these patients experienced a MACE within 30 days (Table 2). Applying a CCS cut-off of 1 or above yielded a sensitivity of $99.5 \%$ (95\% CI 97.3\%-99.9\%) and NPV of 99.1\% (95\% CI 93.9\%-99.9\%). In contrast, there were 722 patients with Ortho hs-cTnI concentrations below $5 \mathrm{ng} / \mathrm{L}$ (68.3\% of the sample); 53 (7.3\%) of these patients experienced a MACE within 30 days. Applying an hs-cTnI cut-off of $5 \mathrm{ng} / \mathrm{L}$ or above yielded a sensitivity of $74.2 \%$ (95\% CI 67.6\%-80.0\%) and NPV of 92.7\% (95\% CI $90.9 \%-94.1 \%)$. Lowering the Ortho hs-cTnI cut-off to $1 \mathrm{ng} / \mathrm{L}$ or above (352 patients with 18 outcomes [5.1\%] for hs-cTnI $<1 \mathrm{ng} / \mathrm{L})$ yielded a sensitivity of $91.2 \%(95 \% \mathrm{CI}$ $86.5 \%-94.7 \%$ ) and NPV of $94.9 \%$ (95\% CI 92.2\%-96.7\%). The specificity for a CCS of 5 was higher than that for hs-cTnI above $11 \mathrm{ng} / \mathrm{L}$ (97.8\%, 95\% CI 96.5\%-98.7\% v. $90.1 \%$, $95 \%$ CI $87.9 \%-92.0 \%)$.

In cohort 2, there were 399 patients with a CCS less than 1 (6.7\% of the sample); fewer than 6 patients had an event at 30 days (exact numbers cannot be reported for privacy reasons per ICES protocol). The point estimates at 30 days for sensitivity/NPV for a CCS cut-off of 1 or above were greater than $99 \%$. By comparison, there were 103 outcomes in 2374 patients $(4.3 \%)$ with Abbott Architect hs-cTnI concentrations less than $5 \mathrm{ng} / \mathrm{L}$. Applying the hs-cTnI cut-off of $5 \mathrm{ng} / \mathrm{L}$ or above yielded a sensitivity of $88.1 \%$ (95\% CI $85.8 \%-90.2 \%)$ and NPV of $95.6 \%$ (95\% CI $94.7 \%-96.4 \%)$. At 90 days, the sensitivity of a CCS cut-off above 1 was $99.3 \%$ (95\% CI 98.6\%-99.7\%), whereas with an hs-cTnI cut-off of $5 \mathrm{ng} / \mathrm{L}$ or above the sensitivity was $87.7 \%$ (95\% CI $85.6 \%-89.6 \%)$. The specificity was higher for a CCS cut-off of 5 than with an hs-cTnI cut-off above $26 \mathrm{ng} / \mathrm{L}(90.0 \%$, 95\% CI $89.1 \%-$ $90.8 \%$ v. $85.5 \%, 95 \%$ CI 84.4-86.4) at 90 days (Table 3 ).

\section{Interpretation}

Patients with a CCS less than 1 for their blood work at ED presentation have an incidence of MACE at 30 days less than or equal to $1 \%$, with a CCS cut-off of 1 or above yielding a sensitivity of at least $99 \%$. Both of these metrics indicate that ED patients with possible ACS who have a CCS less than 1 are a low-risk group of patients, which should enable ED physicians to consider discharge and the cessation of additional investigations in the ED for current and subsequent ACS over the short term. In contrast, patients with a CCS of 5 represent a high-risk group with a specificity of at least $90 \%$, and a higher specificity than using the hs-cTnI 99th percentile cutoffs, which will aid clinicians in triaging patients in the ED to receive additional investigations and care.

With the advent of hs-cTn testing, there have been gains made in the ED for reducing the time between blood draws for testing when evaluating patients with possible ACS.1,34 Also, with the hs-cTn assays, there is evidence to support use 
Table 2: Performance of the clinical chemistry score versus hs-cTnl concentration (measured using the Ortho Vitros $3600 \mathrm{hs}-\mathrm{cTnl}$ assay) alone for identifying risk for 30-day MACE

\begin{tabular}{|c|c|c|}
\hline Score cut-off & $\begin{array}{l}\text { Patients with at least } \\
1 \text { MACE within } 30 \mathrm{~d}\end{array}$ & $\begin{array}{l}\text { Patients with no } \\
\text { MACE within } 30 \mathrm{~d}\end{array}$ \\
\hline CCS $\geq 1$, no. of patients & 204 & 744 \\
\hline CCS $<1$, no. of patients & $1^{*}$ & 109 \\
\hline Sensitivity, \% (95\% Cl) & $99.5(97.3-99.9)$ & \\
\hline Negative predictive value, \% $(95 \% \mathrm{Cl})$ & $99.1(93.9-99.9)$ & \\
\hline Negative likelihood ratio $(95 \% \mathrm{Cl})$ & $0.04(0.01-0.27)$ & \\
\hline Specificity, \% (95\% Cl) & $12.8(10.6-15.2)$ & \\
\hline Positive predictive value, $\%(95 \% \mathrm{Cl})$ & $21.5(21.1-21.9)$ & \\
\hline Positive likelihood ratio $(95 \% \mathrm{Cl})$ & $1.14(1.11-1.17)$ & \\
\hline hs-cTnl $\geq 1 \mathrm{ng} / \mathrm{L}$, no. of patients & 187 & 519 \\
\hline hs-cTnl $<1 \mathrm{ng} / \mathrm{L}$, no. of patients & 18 & 334 \\
\hline Sensitivity, \% (95\% Cl) & $91.2(86.5-94.7)$ & \\
\hline Negative predictive value, \% $(95 \% \mathrm{Cl})$ & $94.9(92.2-96.7)$ & \\
\hline Negative likelihood ratio $(95 \% \mathrm{Cl})$ & $0.22(0.14-0.35)$ & \\
\hline Specificity, \% (95\% Cl) & $39.2(35.9-42.5)$ & \\
\hline Positive predictive value, \% $(95 \% \mathrm{Cl})$ & $26.5(25.2-27.8)$ & \\
\hline Positive likelihood ratio $(95 \% \mathrm{Cl})$ & $1.50(1.40-1.61)$ & \\
\hline hs-cTnl $\geq 5$ ng/L, no. of patients & 152 & 183 \\
\hline hs-cTnl $<5$ ng/L, no. of patients & 53 & 669 \\
\hline Sensitivity, \% (95\% Cl) & $74.2(67.6-80.0)$ & \\
\hline Negative predictive value, \% $(95 \% \mathrm{Cl})$ & $92.7(90.9-94.1)$ & \\
\hline Negative likelihood ratio $(95 \% \mathrm{Cl})$ & $0.33(0.26-0.42)$ & \\
\hline Specificity, \% (95\% Cl) & $78.5(75.6-81.2)$ & \\
\hline Positive predictive value, \% $(95 \% \mathrm{Cl})$ & $45.4(41.6-49.1)$ & \\
\hline Positive likelihood ratio $(95 \% \mathrm{Cl})$ & $3.45(2.97-4.02)$ & \\
\hline CCS $=5$, no. of patients & 58 & 19 \\
\hline CCS $<5$, no. of patients & 147 & 834 \\
\hline Specificity, \% (95\% Cl) & $97.8(96.5-98.7)$ & \\
\hline Positive predictive value, \% $(95 \% \mathrm{Cl})$ & $75.3(65.0-83.4)$ & \\
\hline Positive likelihood ratio (95\% Cl) & $12.7(7.74-20.8)$ & \\
\hline Sensitivity, \% (95\% Cl) & $28.3(22.2-34.9)$ & \\
\hline Negative predictive value, $\%(95 \% \mathrm{Cl})$ & $85.0(83.9-86.1)$ & \\
\hline Negative likelihood ratio $(95 \% \mathrm{Cl})$ & $0.73(0.67-0.80)$ & \\
\hline hs-cTnl > $11 \mathrm{ng} / \mathrm{L}$, no. of patients & 132 & 84 \\
\hline hs-cTnl $\leq 11 \mathrm{ng} / \mathrm{L}$, no. of patients & 73 & 768 \\
\hline Specificity, \% (95\% Cl) & $90.1(87.9-92.0)$ & \\
\hline Positive predictive value, $\%(95 \% \mathrm{Cl})$ & $61.1(55.6-66.4)$ & \\
\hline Positive likelihood ratio $(95 \% \mathrm{Cl})$ & $6.5(5.2-8.2)$ & \\
\hline Sensitivity, \% (95\% Cl) & $64.4(57.4-70.9)$ & \\
\hline Negative predictive value, $\%(95 \% \mathrm{Cl})$ & $91.3(89.7-92.7)$ & \\
\hline Negative likelihood ratio $(95 \% \mathrm{Cl})$ & $0.39(0.33-0.47)$ & \\
\hline \multicolumn{3}{|c|}{$\begin{array}{l}\text { Note: } \mathrm{CCS}=\text { clinical chemistry score, } \mathrm{Cl}=\text { confidence interval, hs-cTNI }=\text { high-sensitivity cardiac troponin I, hs-cTNT = } \\
\text { high-sensitivity cardiac troponin T, MACE = major adverse cardiac event. } \\
\text { *At presentation, this patient had the following values: } \mathrm{hs}-\mathrm{Tnl}<1 \mathrm{ng} / \mathrm{L} \text { with the Ortho assay, hs-cTnl }<2 \mathrm{ng} / \mathrm{L} \text { with the Abbott } \\
\text { assay and hs-cTnT }<3 \mathrm{ng} / \mathrm{L} \text { with the Roche assay. The patient's } 30 \text {-day outcome was coronary artery bypass grafting. }\end{array}$} \\
\hline
\end{tabular}




\begin{tabular}{|c|c|c|}
\hline Score cut-off & $\begin{array}{l}\text { Patients with at least } \\
1 \text { MACE within } 90 \mathrm{~d}\end{array}$ & $\begin{array}{l}\text { Patients with no } \\
\text { MACE within } 90 \mathrm{~d}\end{array}$ \\
\hline $\operatorname{CCS} \geq 1$, no. of patients & 1092 & 4483 \\
\hline CCS $<1$, no. of patients ${ }^{*}$ & 8 & 391 \\
\hline Sensitivity, \% (95\% Cl) & $99.3(98.6-99.7)$ & \\
\hline Negative predictive value, $\%(95 \% \mathrm{Cl})$ & $98.0(96.1-99.0)$ & \\
\hline Negative likelihood ratio $(95 \% \mathrm{Cl})$ & $0.09(0.05-0.18)$ & \\
\hline Specificity, \% (95\% Cl) & $8.0(7.3-8.8)$ & \\
\hline Positive predictive value, $\%(95 \% \mathrm{Cl})$ & $19.6(19.4-19.7)$ & \\
\hline Positive likelihood ratio $(95 \% \mathrm{Cl})$ & $1.08(1.07-1.09)$ & \\
\hline hs-cTnl $\geq 5 \mathrm{ng} / \mathrm{L}$, no. of patients & 965 & 2635 \\
\hline hs-cTnl $<5$ ng/L, no. of patients & 135 & 2239 \\
\hline Sensitivity, \% (95\% Cl) & $87.7(85.6-89.6)$ & \\
\hline Negative predictive value, \% $(95 \% \mathrm{Cl})$ & $94.3(93.4-95.1)$ & \\
\hline Negative likelihood ratio $(95 \% \mathrm{Cl})$ & $0.27(0.23-0.31)$ & \\
\hline Specificity, \% (95\% Cl) & $45.9(44.5-47.3)$ & \\
\hline Positive predictive value, $\%(95 \% \mathrm{Cl})$ & $26.8(26.1-27.5)$ & \\
\hline Positive likelihood ratio $(95 \% \mathrm{Cl})$ & $1.62(1.57-1.68)$ & \\
\hline $\mathrm{CCS}=5$, no. of patients & 420 & 488 \\
\hline CCS $<5$, no. of patients & 680 & 4386 \\
\hline Specificity, \% (95\% Cl) & $90.0(89.1-90.8)$ & \\
\hline Positive predictive value, $\%(95 \% \mathrm{Cl})$ & $46.3(43.5-49.1)$ & \\
\hline Positive likelihood ratio $(95 \% \mathrm{Cl})$ & $3.81(3.41-4.27)$ & \\
\hline Sensitivity, \% (95\% Cl) & $38.2(35.3-41.1)$ & \\
\hline Negative predictive value, $\%(95 \% \mathrm{Cl})$ & $86.6(86.0-87.1)$ & \\
\hline Negative likelihood ratio $(95 \% \mathrm{Cl})$ & $0.69(0.66-0.72)$ & \\
\hline hs-cTnl > $26 \mathrm{ng} / \mathrm{L}$, no. of patients & 567 & 709 \\
\hline hs-cTnl $\leq 26 \mathrm{ng} / \mathrm{L}$, no. of patients & 533 & 4165 \\
\hline Specificity, \% (95\% Cl) & $85.5(84.4-86.4)$ & \\
\hline Positive predictive value, $\%(95 \% \mathrm{Cl})$ & $44.4(42.2-46.6)$ & \\
\hline Positive likelihood ratio $(95 \% \mathrm{Cl})$ & $3.54(3.24-3.87)$ & \\
\hline Sensitivity, \% (95\% Cl) & $51.6(48.5-54.5)$ & \\
\hline Negative predictive value, $\%(95 \% \mathrm{Cl})$ & 88.7 (88.0-89.3) & \\
\hline Negative likelihood ratio $(95 \% \mathrm{Cl})$ & $0.57(0.53-0.60)$ & \\
\hline \multicolumn{3}{|c|}{$\begin{array}{l}\text { Note: } \mathrm{CCS}=\text { clinical chemistry score, } \mathrm{Cl}=\text { confidence interval, hs-cTnl = high-sensitivity cardiac troponin I, MACE = major } \\
\text { adverse cardiac event. } \\
\text { *At } 30 \mathrm{~d} \text {, MACE occurred in } \leq 5 \text { patients with a CCS }<1 \text { and these results cannot be reported per ICES standard operating } \\
\text { procedures where counts }<6 \text { are suppressed for privacy reasons. }\end{array}$} \\
\hline
\end{tabular}

of a low hs-cTn concentration to rule out MI at ED presentation. ${ }^{1-6}$ However, there are important caveats to using a low hs-cTnI concentration alone for decision-making in the ED: patients who present early after pain onset may be misdiagnosed; ruling out $\mathrm{MI}$ is not equivalent to clearing a patient for discharge as patients may still be at risk for ischemic events; and analytical imprecision at the low end for hs-cTn assays may result in patient misclassification. 4,35,36 Moreover, in an attempt to simplify interpretation of a low hs-cTn concentration, some have advocated for and studies have assessed a single cut-off of less than $5 \mathrm{ng} / \mathrm{L}$. ${ }^{2,3,6,31}$

This approach may be suitable for hs-cTnI assays if there is close agreement with the Abbott hs-c'TnI assay at this cut-off, as this assay has been assessed in large multicentre studies assessing MI. ${ }^{3,23}$ However, our findings indicate that the $5 \mathrm{ng} / \mathrm{L}$ cut-off alone at presentation for the Ortho hs-cTnI assay would be unsafe, with over $7 \%$ of patients with hs-cTnI concentrations less than $5 \mathrm{ng} / \mathrm{L}$ having MACE with a sensitivity below $75 \%$ at 
this cut-off. Lowering the hs-c $\mathrm{TnI}$ threshold to $1 \mathrm{ng} / \mathrm{L}$ as used in the 0-hour and 1-hour algorithm with the Ortho hs-cTnI assay yields sensitivity (91\%) close to that of the Abbott hs-cTnI assay when using the $5 \mathrm{ng} / \mathrm{L}$ cut-off (88\%). Importantly, both estimates by these different hs-cTnI assays would be too low to exclude MACE. Only the CCS cut-off of 1 or above with both assays in 2 different populations obtained the required sensitivity $(\geq 99 \%)$ to rule out MACE at 30 days.

Future studies will need to assess performance of the CCS with different blood sample types and to determine whether different combinations of point-of-care testing with the core chemistry analyzers can achieve acceptable clinical performance.

\section{Limitations}

As with any observational study, there are limitations. Because ED physicians were not exposed to the hs-cTnI results or the CCS, the impact on patient care of applying either the hs-cTnI cut-off of $5 \mathrm{ng} / \mathrm{L}$ or above or the CCS cut-off of 1 or above cannot be assessed. The 2 cohorts in this study shared a common variable in that both consisted of patients treated at the same hospitals in Hamilton (although over different time periods); as such, the impact of different geographic locations on the CCS cannot be assessed. However, previous studies across different countries and across different provinces in Canada have demonstrated close agreement of the CCS in different geographic locations and using different hs-cTn assays. ${ }^{10,37}$

As the Ortho Vitros 3600 hs-cTnI assay was done using samples collected in 2013, there might have been some degradation in analyte; since it is the latest hs-cTnI assay approved by Health Canada, real-world analytic precision data for this assay are not available. However, there are data to support the long-term stability of the cardiac troponin I analyte as measured by the Ortho Vitros 3600 hs-cTnI assay in samples stored frozen for over 15 years, ${ }^{28}$ with the assay achieving a precision of $10 \%$ or less at normal concentrations and thus fulfilling the analytic requirements for an hs-c $\mathrm{TnI}$ assay. ${ }^{14,15}$

The 2 cohorts had outcomes assessed differently (i.e., adjudication v. databases) and different lengths of follow-up (although the 30-d outcomes obtained for both cohorts are of importance to ED physicians), ${ }^{7}$ which prevents us from combining these findings. Differences in outcome classifications between the 2 cohorts are an important limitation; however, administrative databases, as used in cohort 2, can be a robust source..$^{38}$ Finally, additional approaches, such as serial testing with hs-cTn, may further identify patients at low risk and suitable for discharge; however, ED patients with a CCS less than 1 at presentation have a lower mortality incidence at 1 year than ED patients with an hs-cTnI concentration less than $5 \mathrm{ng} / \mathrm{L}$ at presentation ( $2 \% \mathrm{v} .5 \%$, respectively). ${ }^{20}$

\section{Conclusion}

A CCS less than 1 and 5 with 2 different hs-c TnI assays identified patients at low and high risk for MACE at 30 days, respectively; this performance cannot be achieved when using hscTnI testing alone. An intervention study is needed to evaluate the impact of the CCS at both the patient and hospital levels.

\section{References}

1. Roffi M, Patrono C, Collet JP, et al. 2015 ESC guidelines for the management of acute coronary syndromes in patients presenting without persistent ST-segment elevation: Task Force for the Management of Acute Coronary Syndromes in Patients Presenting without Persistent ST-Segment Elevation of the European Society of Cardiology (ESC). Eur Heart 7 2016;37:267-315.

2. Pickering JW, Than MP, Cullen L, et al. Rapid rule-out of acute myocardial infarction with a single high-sensitivity cardiac troponin $\mathrm{T}$ measurement below the limit of detection: a collaborative meta-analysis. Ann Intern Med 2017;166:715-24.

3. Chapman AR, Lee KK, McAllister DA, et al. Association of high-sensitivity cardiac troponin I concentration with cardiac outcomes in patients with suspected acute coronary syndrome. [published erratum in fAMA 2018;319:1168.] 7AMA 2017;318:1913-24.

4. Thygesen K, Alpert JS, Jaffe AS, et al.; ESC Scientific Document Group. Fourth universal definition of myocardial infarction (2018). Eur Heart 7 2019;40:237-69.

5. Greenslade J, Cho E, Van Hise C, et al. Evaluating rapid rule-out of acute myocardial infarction using a high-sensitivity cardiac troponin I assay at presentation. Clin Chem 2018;64:820-9.

6. Sandoval Y, Nowak R, deFilippi CR, et al. Myocardial infarction risk stratification with a single measurement of high-sensitivity troponin I. 7 Am Coll Cardiol 2019;74:271-82.

7. Than M, Herbert M, Flaws D, et al. What is an acceptable risk of major adverse cardiac event in chest pain patients soon after discharge from the emergency department? A clinical survey. Int 7 Cardiol 2013;166:752-4.

8. American College of Emergency Physicians Clinical Policies Subcommittee (Writing Committee) on Suspected Non-ST-Elevation Acute Coronary Syndromes; Tomaszewski CA, Nestler D, Shah KH, et al. Clinical policy: critical issues in the evaluation and management of emergency department patients with suspected non-ST-elevation acute coronary syndromes. Ann Emerg Med 2018;72:e65-106.

9. Peacock WF, Baumann BM, Bruton D, et al. Efficacy of high-sensitivity troponin $\mathrm{T}$ in identifying very-low-risk patients with possible acute coronary syndrome. 7AMA Cardiol 2018;3:104-11.

10. Kavsak PA, Neumann JT, Cullen L, et al. Clinical chemistry score versus high-sensitivity cardiac troponin $\mathrm{I}$ and $\mathrm{T}$ tests alone to identify patients at low or high risk for myocardial infarction or death at presentation to the emergency department. CMA7 2018;190:E974-84.

11. Raizman JE, Fuezery A, Tsui AKY, et al. Multicenter comparison of imprecision at low concentrations of two regulatory approved high-sensitivity cardiac troponin I assays. Clin Chim Acta 2018;486:219-20.

12. Kavsak PA, Worster A, Hill SA, et al. Evaluation of the Siemens ADVIA Centaur high-sensitivity cardiac troponin I assay in serum. Clin Chim Acta 2018;487:216-21.

13. Kavsak PA, MacCuish J, Boreyko J, et al. Analytical characterization of the Siemens Dimension EXL high-sensitivity cardiac troponin I assay. Clin Biochem 2019;69:52-6.

14. Kavsak PA, Mondoux SE, Sherbino J, et al. Clinical evaluation of Ortho Clinical Diagnostics high-sensitivity cardiac troponin I assay in patients with symptoms suggestive of acute coronary syndrome. Clin Biochem 2020;80: 48-51.

15. Wu AHB, Christenson R, Greene D, et al. Clinical laboratory practice recommendations for the use of cardiac troponin in acute coronary syndrome: expert opinion from the Academy of the American Association for Clinical Chemistry and the Task Force on Clinical Applications of Cardiac BiolMarkers of the International Federation of Clinical Chemistry and Laboratory Medicine. Clin Chem 2018;64:645-55.

16. Kavsak PA, Ainsworth C, Clark L, et al. Four different high-sensitivity cardiac troponin assays with important analytical performance differences. Can 7 Cardiol 2019;35:796.e17-8.

17. Kavsak PA. Early standardization of high sensitivity troponin T reporting a lost opportunity. Clin Biochem 2011;44:758-9.

18. Lam L, Aspin L, Heron RC, et al. Discrepancy between cardiac troponin assays due to endogenous antibodies. Clin Chem 2020;66:445-54.

19. Kavsak PA, Tandon V, Ainsworth C. A Three-site immunoassay for highsensitivity cardiac troponin I with low immunoreactivity for macrocomplexes. Clin Chem 2020;66:854-5.

20. Kavsak PA, Cerasuolo JO, Ko DT, et al. High-sensitivity cardiac troponin I versus a clinical chemistry score for predicting all-cause mortality in an emergency department population. CFC Open 2020;2:296-302.

21. Shortt C, Ma J, Clayton N, et al. Rule-in and rule-out of myocardial infarction using cardiac troponin and glycemic biomarkers in patients with symptoms suggestive of acute coronary syndrome. Clin Chem 2017;63:403-14.

22. Kavsak PA, Worster A, Ma J, et al. High-sensitivity cardiac troponin risk cutoffs for acute cardiac outcomes at emergency department presentation. Can $\mathcal{7}$ Cardiol 2017;33:898-903.

23. Neumann JT, Twerenbold R, Ojeda F, et al. Application of high-sensitivity troponin in suspected myocardial infarction. N Engl f Med 2019;380:2529-40.

24. Kavsak PA, Shortt C, Pond G, et al. High-sensitivity cardiac troponin I for predicting death in a female emergency department population. Clin Chem 2014;60:271-3. 
25. Lau G, Koh M, Kavsak PA, et al. Clinical outcomes for chest pain patients discharged home from emergency departments using high-sensitivity versus conventional cardiac troponin assays. Am Heart 7 2020;221:84-94.

26. Kavsak PA, Roy C, Malinowski P, et al. Sample matrix and high-sensitivity cardiac troponin I assays. Clin Chem Lab Med 2019;57:745-51.

27. Kavsak PA, Petryayeva E, Clark L. Analytical variation and Abbott Diagnostics high-sensitivity cardiac troponin I risk categories in asymptomatic individuals. Can 7 Cardiol 2019;35:1605.e7-8.

28. Kavsak PA, Clark L, Caruso N, et al. Macrocomplexes and high-sensitivity cardiac troponin assays in samples stored for over 15 years. Clin Chim Acta 2020; 505:6-8.

29. Collinson PO, Saenger AK, Apple FS, et al. High sensitivity, contemporary and point-of-care cardiac troponin assays: educational aids developed by the IFCC Committee on Clinical Application of Cardiac Bio-Markers. Clin Chem Lab Med 2019;57:623-32.

30. Levey AS, Stevens LA, Schmid CH, et al. A new equation to estimate glomerular filtration rate. Ann Intern Med 2009;150:604-12.

31. Eggers KM, Jernberg T, Ljung L, et al. High-sensitivity cardiac troponin-based strategies for the assessment of chest pain patients - a review of validation and clinical implementation studies. Clin Chem 2018;64:1572-85.

32. Boeddinghaus J, Twerenbold R, Nestelberger T, et al. Clinical use of a new high-sensitivity cardiac troponin I assay in patients with suspected myocardial infarction. Clin Chem 2019;65:1426-36.

33. Apple FS, Wu AH, Sandoval Y, et al. Sex-specific 99th percentile upper reference limits for high sensitivity cardiac troponin assays derived using a universal sample bank. Clin Chem 2020;66:434-44.

34. Kufaishi H, Pardhan A, Krizmanich W, et al. Adopting 'ng/L' as the units for high-sensitivity cardiac troponin assays and commitment by the entire health-care team could be the key for adopting recommendations. Ann Clin Biochem 2016;53:516-7.

35. Lyon AW, Kavsak PA, Lyon OA, et al. Simulation models of misclassification error for single thresholds of high-sensitivity cardiac troponin I due to assay bias and imprecision. Clin Chem 2017;63:585-92.

36. Kavsak PA. External quality assessment testing near the limit of detection for high-sensitivity cardiac troponin assays. Clin Chem 2018;64:1402-4.

37. Kavsak PA, McRae A, Vatanpour S, et al. A multicenter assessment of the sensitivity and specificity for a single high-sensitivity cardiac troponin test at emergency department presentation for hospital admission. 7 Appl Lab Med 2019;4:170-9.

38. Meah MN, Denvir MA, Mills NL, et al. Clinical endpoint adjudication. Lancet 2020;395:1878-82.

Affiliations: Department of Pathology and Molecular Medicine (Kavsak, Hill, McQueen), McMaster University; ICES McMaster (Cerasuolo, Perez, Seow), Faculty of Health Sciences, McMaster University, Hamilton, Ont.; ICES (Ko), Toronto, Ont.; Department of Health Research Methods, Evidence, and Impact (Ma, Griffith); Division of Emergency Medicine
(Sherbino, Mondoux, Worster); Department of Medicine (Clayton); Division of Cardiology, and Population Health Research Institute (Mehta, Devereaux), McMaster University, Hamilton, Ont.

Contributors: Peter Kavsak, Andrew Worster and Dennis Ko were responsible for concept and design. All authors acquired, analyzed or interpreted the data. Peter Kavsak and Andrew Worster drafted the manuscript. Joshua Cerasuolo, Dennis Ko, Jinhui Ma, Jonathan Sherbino, Shawn Mondoux, Natasha Clayton, Stephen Hill, Matthew McQueen, Lauren Griffith, Shamir Mehta, Richard Perez, Hsien Seow and P.J. Devereaux critically revised the manuscript for important intellectual content. All of the authors gave final approval of the version to be published and agreed to be accountable for all aspects of the work.

Funding: This study was supported by a Canadian Institutes of Health Research grant (funding reference no. 155964), with funding by Ortho Clinical Diagnostics to perform high-sensitivity cardiac troponin I testing in cohort 1 (Optimum Troponin Cutoffs for ACS in the ED [ROMI-3] study; ClinicalTrials.gov identifier no. NCT01994577) and by Abbott Laboratories to perform high-sensitivity cardiac troponin I testing in cohort 2.

Data sharing: The studies were conducted before data-sharing processes were in place, and thus individual data are not available.

Acknowledgements: The authors thank IMS Brogan for use of their Drug Information Database (for cohort 2). Special thanks to the staff at the Clinical Research Laboratory and Biobank in Hamilton, Ontario, for the storage, management and testing of the samples as well as to Dr. Saranya Arnoldo for provision of some study materials.

Disclaimer: This study was supported by ICES, which is funded by an annual grant from the Ontario Ministry of Health and Long-Term Care (MOHLTC). The opinions, results and conclusions reported in this article are those of the authors and are independent from the funding sources. No endorsement by ICES or the Ontario MOHLTC is intended or should be inferred. This study uses data provided by the Canadian Institute for Health Information (CIHI). Analyses, opinions and statements expressed herein are those of the authors and not necessarily those of CIHI; no endorsement is intended or should be inferred.

Supplemental information: For reviewer comments and the original submission of this manuscript, please see www.cmajopen.ca/content/8/4/ E676/suppl/DC1. 